\title{
QUALITATIVE EVALUATION OF ANTIBIOTICS FROM VARIOUS WATER RESOURCES IN AND AROUND PERUNGUDI DUMPSITE, CHENNAI
}

\author{
Sija Arun ${ }^{1, *}$, S. Krithiga ${ }^{1}$ and Paromita Chakraborty ${ }^{2}$ \\ ${ }^{1}$ Department of Civil Engineering, SRM Institute of Science and Technology, \\ Kattankulathur -603203, Tamil Nadu, India \\ ${ }^{2}$ SRM Research Institute, SRM Institute of Science and Technology, \\ Kattankulathur -603203, Tamil Nadu, India \\ *E-mail:sijaarun08@gmail.com
}

\begin{abstract}
India, a country having a population of 1.27 billion is considered as the eleventh biggest consumer country in the world. Due to globalization, a large variety of antibiotics are entering into the Indian market and subsequently, they reach natural resources. Most of the Indian cities are located closer to natural resources and the contaminants get discharged into the environment with or without treatment. Conventional treatment methods in the wastewater treatment plant do not remove antibiotics. This necessitates a deeper analysis and study on the occurrence of antibiotics in the water resources and treatment plants in India. This study is aimed to qualitatively detect the occurrence of four common antibiotics, viz., Amoxicillin, Erythromycin, Cefixime, and Azithromycin from a wastewater treatment plant at Perungudi and some of the water resources near to the wastewater treatment plant (WWTP). Samples were collected and extracted by using solid-phase extraction (SPE). Analysis of the extracted samples was done in High-Performance Liquid Chromatography (HPLC) to determine the presence of the four antibiotics. Amoxicillin was detected in all the water samples. WWTP influent and effluent showed the presence of Amoxicillin, which indicates that the treatment methods in the plant do not fully remove this contaminant. Cefixime which was detected only in WWTP influent, indicating that it is completely removed in the treatment plant. Erythromycin, Azithromycin, and Amoxicillin were detected in municipality water, which shows that surface washings from the landfill site are reaching the water resources and the water treatment methods do not fully remove these contaminants.
\end{abstract}

Keywords: Antibiotics, Solid Phase Extraction, HPLC.

@ RASĀYAN. All rights reserved

\section{INTRODUCTION}

Pharmaceuticals especially antibiotics play a vital role in our daily lives for the treatment of various diseases. Antibiotics play a critical role in human health and animal growth promoters, but the scientific community was unaware of its release into the environment till late 1990. The aquatic environment is contaminated with antibiotics in different ways. Once the drug is administered to humans, the unmetabolized part of the drug is discharged through excreta and it reaches the sewage treatment plant. There are greater chances of introduction of this contaminant into the environment by runoff and groundwater contamination from confined animal feeding operations ${ }^{1}$.

The conventional treatment plants are not designed to treat the antibiotic residues. So, the treatment plant effluent may release the residues from the plant to natural resources. The unused drugs and expired drugs are disposed of improperly to solid waste dumping sites. During the rainy season the antibiotic residues washed away with rainwater and reach natural resources like river and lakes. Some part of the compound leach through the soil and contaminate ground water also. The surface and ground water are used by the people for many purposes. The municipality authorities also depend on surface and groundwater for their source of water supply. Even if treatments are given to the water to meet the drinking water standards, there is no particular treatment method to remove this kind of micro contaminants from water in a water treatment

Rasayan J. Chem., 12(2), 915-920(2019)

http://dx.doi.org/10.31788/RJC.2019.1225161

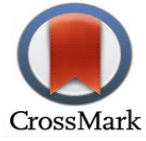


plant facility. In total, a cocktail of antibiotic residue reaches the human body, and generates antibiotic resistance which has emerged as a global issue during this period.

A lot of studies have been conducted worldwide to determine the occurrence of antibiotics in wastewater treatment plant as well as natural resources. These studies show that antibiotics are present in river water at nanogram level and sewage treatment does not completely remove the antibiotic from water ${ }^{3,4}$. The objective of this study is to qualitatively determine the occurrence of different types of antibiotics in a treatment plant and in water sources around the treatment plant facility.

\section{EXPERIMENTAL}

Material and Methods

A survey has been conducted with the pharmacies in the areas such as Guindy, Velachery, Gandhinagar, Indiranagar which are the areas around Perungudi for the selection of antibiotics and it has been found that Azithromycin, Cefixime, Erythromycin and Amoxicillin are the commonly prescribed and sold antibiotics in the Perungudi area. The physicochemical properties of the compounds (Table-1).

Table-1: Physiochemical Properties of Selected Antibiotics.

\begin{tabular}{c|c|c|c}
\hline Antibiotics & Formula & $\begin{array}{c}\text { Molecular weight } \\
\text { gmol }\end{array}$ & Antibiotic Class \\
\hline Azithromycin & $\mathrm{C}_{38} \mathrm{H}_{12} \mathrm{~N}_{2} \mathrm{O}_{12}$ & 748.984 & Macrolide \\
\hline Cefixime & $\mathrm{C}_{38} \mathrm{H}_{15} \mathrm{~N}_{5} \mathrm{O}_{7} \mathrm{~S}_{2}$ & 453.452 & Cephalosporin \\
\hline Erythromycin & $\mathrm{C}_{37} \mathrm{H}_{67} \mathrm{NO}_{13}$ & 733.93 & Macrolide \\
\hline Amoxicillin & $\mathrm{C}_{16} \mathrm{H}_{19} \mathrm{~N}_{3} \mathrm{O}_{5} \mathrm{~S}$ & 365.4 & $\beta$-lactam \\
\hline
\end{tabular}

The chemical structure and pictorial representation of the selected antibiotics are shown in Fig.-1.<smiles>CC1(C)S[C@@H]2[C@H](NC(=O)[C@@H](N)c3ccc(O)cc3)C(=O)N2[C@H]1C(=O)O</smiles><smiles>Nc1nc(/C(=N/OCC(=O)O)C(=O)N[C@H]2C(=O)N3C(C(=O)O)=C(/C=C/C=O)CS[C@H]23)cs1</smiles>

Amoxicillin

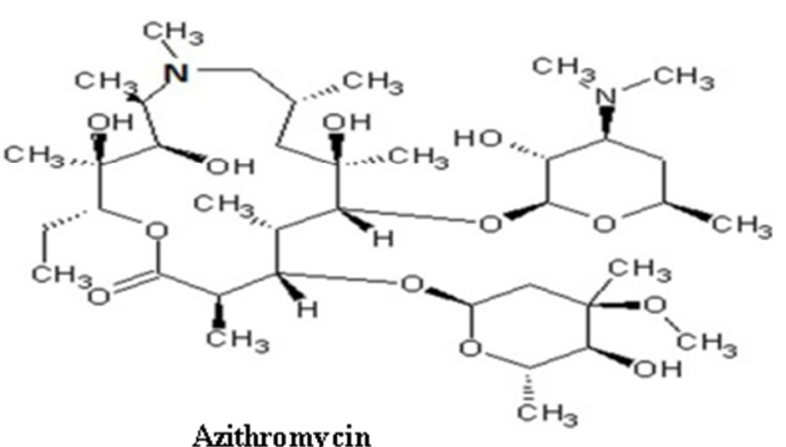

Cefixime

Aathromycin

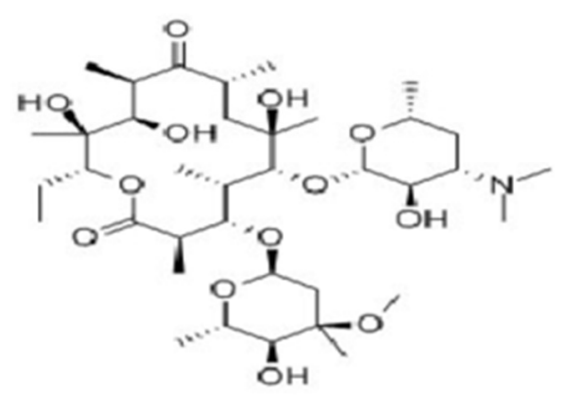

Erythromycin

Fig.-1: Chemical Structure of Antibiotics

The antibiotic standards were bought from Sigma-Aldrich and a stock solution of $1000 \mathrm{ppm}$ was prepared. The working solutions were prepared from the stock solution which is having concentrations of $500 \mathrm{ppm}$, $250 \mathrm{ppm}, 100 \mathrm{ppm}$ and $50 \mathrm{ppm}$. All the stock solutions were prepared by using methanol. The standard 
solutions were injected in HPLC and then followed by the samples to qualitatively detect whether the antibiotics are present in the sample.

\section{Sample Collection and Preparation}

A total of 5 samples were collected from different sources around the Perungudi wastewater treatment plant. The sampling points were as follows i.e., groundwater source, Perungudi lake, municipality drinking water sample, inlet and outlet sample from Perungudi wastewater treatment plant. The samples were collected in 1 litre amber colored bottles. The bottles were rinsed with methanol and milli pore water prior to the collection of samples. Samples collected were tested for $\mathrm{pH}$ and reduce the $\mathrm{pH}$ to 3 using $4 \mathrm{M}$ concentrated $\mathrm{H}_{2} \mathrm{SO}_{4}$ for better ion exchange during solid phase extraction. The samples were then filtered through a 0.45 $\mu \mathrm{m}$ glass membrane filter to remove all suspended impurities.

\section{Solid-Phase Extraction}

A solid-phase extraction apparatus was used to extract the sample after acidification and filtration. An anion-exchange HLB cartridge was used in the solid phase extraction. The cartridges were conditioned first with methanol and then with milli-Q water, prior to extraction. The sample was allowed to pass through the cartridge under vacuum at a flow rate of $5 \mathrm{ml} / \mathrm{min}$. After loading of samples, the cartridges were allowed to dry for one day and then eluted with $20 \mathrm{ml}$ acidified methanol. A pinch of anhydrous sodium sulphate was added for dehydration. The extracted samples were analyzed inHigh Performance Liquid Chromatography (HPLC) with UV detector.

\section{High-Performance Liquid Chromatography}

Following are the settings for the determination of the four antibiotics in HPLC. The column used was XDB C18 column $250 \times 4.6 \mathrm{~mm}$ id, $5 \mu \mathrm{m}$ particle size. The flow rate was $0.6 \mathrm{ml} / \mathrm{minute}$. For azithromycin and erythromycin, mobile phase $\mathrm{A}$ is $90 \%$ water with $10 \%$ ammonium acetate, mobile phase $\mathrm{B}$ is $90 \%$ acetonitrile and $10 \%$ methanol. For amoxicillin, mobile phase A was water with $0.2 \%$ formic acid, mobile phase B was HPLC grade methanol with 2\% HPLC grade formic acid. Flow rate was $0.6 \mathrm{ml} / \mathrm{min}$ with $80 \%$ of A and $20 \%$ of B. For cefixime, mobile phase A includes water with $0.5 \%$ formic acid and mobile phase B was acetonitrile with $0.5 \%$ formic acid. Calibration graph for all the antibiotics were done with 5 trials (1000ppm, 500ppm, 250ppm, 100ppm, 50ppm) where linearity was identified ${ }^{15}$.

\section{Chromatogram and Retention Time of Standards}

\section{RESULTS AND DISCUSSION}

As a first procedure, all four standards were injected into HPLC following the conditions provided for each compound. The retention time of all the standards was detected within the first seven minutes. Chromatogram of all the standards azithromycin, erythromycin, amoxicillin and cefixime are shown in figure-2. The retention time of azithromycin is 3.55 minutes and that of erythromycin, amoxicillin and cefixime are 3.52, 3.79 and 5.24 respectively.

From the above-obtained chromatogram diagrams, the retention time for the standards azithromycin, erythromycin, amoxicillin and cefixime were obtained and their presence in various samples was detected by calculating plus or minus $2.5 \%$ of the retention time and the details of the retention time are shown Table-2.

Table-2: Retention Time of Standards

\begin{tabular}{c|c|c|c}
\hline Antibiotics & $\begin{array}{c}\text { Retention } \\
\text { Time(min) }\end{array}$ & $\begin{array}{c}\text { Retention Time } \\
(+2.5 \%)\end{array}$ & $\begin{array}{c}\text { Retention Time (- } \\
2.5 \%)\end{array}$ \\
\hline Azithromycin & 3.55 & 3.64 & 3.46 \\
\hline Erythromycin & 3.52 & 3.61 & 3.43 \\
\hline Amoxicillin & 3.79 & 3.88 & 3.69 \\
\hline Cefixime & 5.24 & 5.38 & 5.12 \\
\hline
\end{tabular}

By comparing the retention time obtained from standards and the samples, the occurrence of antibiotics compounds were detected. Table-3 shows the occurrence of investigated antibiotic compounds in the samples. 
RASĀYAN J. Chem.

Vol. 12 | No. 2 |915 - 920| April - June | 2019
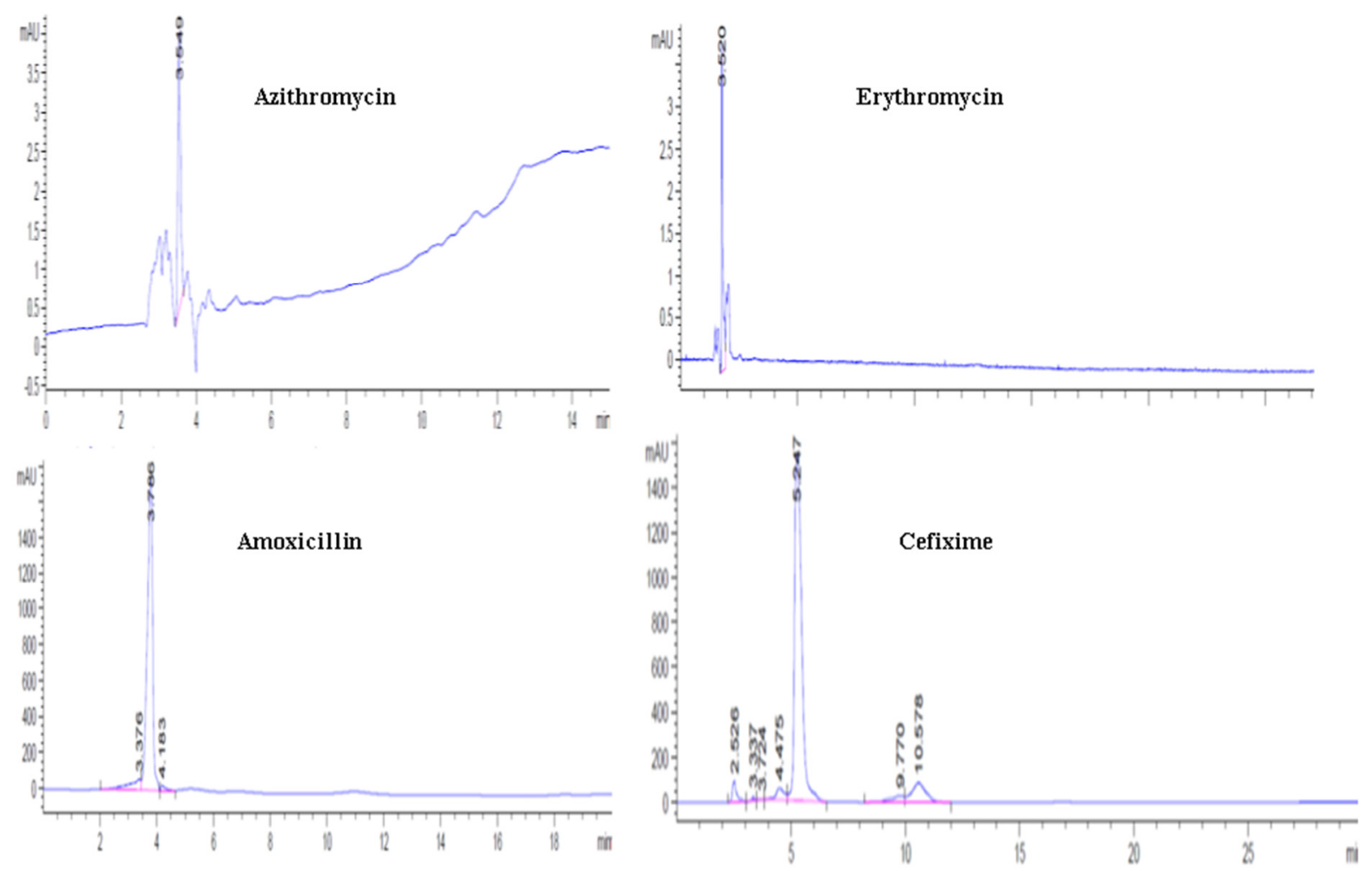

Fig.-2: Chromatogram of Standards

Table-3 Occurrence of Selected Antibiotics in Samples

\begin{tabular}{c|c|c|c|c|c|c}
\hline Compound & $\begin{array}{c}\text { Ground } \\
\text { water }\end{array}$ & $\begin{array}{c}\text { Treatment } \\
\text { plant-inlet }\end{array}$ & $\begin{array}{c}\text { Treatment } \\
\text { plant-outlet }\end{array}$ & lake & $\begin{array}{c}\text { Municipality } \\
\text { water }\end{array}$ & Marshland \\
\hline Azithromycin & $\mathrm{ND}^{1}$ & $\mathrm{D}$ & $\mathrm{ND}$ & $\mathrm{ND}$ & $\mathrm{D}$ & $\mathrm{ND}$ \\
\hline Erythromycin & $\mathrm{ND}$ & $\mathrm{ND}$ & $\mathrm{ND}$ & $\mathrm{ND}$ & $\mathrm{D}$ & $\mathrm{ND}$ \\
\hline Amoxicillin & $\mathrm{D}^{2}$ & $\mathrm{D}$ & $\mathrm{D}$ & $\mathrm{D}$ & $\mathrm{D}$ & $\mathrm{D}$ \\
\hline Cefixime & $\mathrm{ND}$ & $\mathrm{D}$ & $\mathrm{ND}$ & $\mathrm{ND}$ & $\mathrm{ND}$ & $\mathrm{ND}$ \\
\hline
\end{tabular}

1-Not detected, 2- Detected

Amoxicillin is detected in all the samples. The chromatogram of amoxicillin in various samples is shown in figure-3. The chromatogram for amoxicillin in municipality water shows a lot of interferences, so the confirmation of presence compound in the sample cannot be done accurately. Amoxicillin is detected in the inlet and outlet of WWTP. Studies from Delhi treatment plant also reported the occurrence of amoxicillin in inlet and outlet of WWTP ${ }^{11}$. Azithromycin and cefixime show its presence in inlet water sample of WWTP and it is absent in the outlet sample of WWTP. It indicates that these two compounds are completely removed in wastewater treatment processes. Out of the four compounds, except cefixime, all other compounds are present in the municipality drinking water. This indicates that the source of water that the city depends on its drinking water supply is contaminated with pharmaceuticals. The main sources of drinking water supply inside the city are lakes. These lakes are not protected properly. People have a habit of throwing the waste materials into the water bodies. So, there are chances of throwing unused medicines into these lakes. And the water treatment methods do not have the facilities to treat these micro contaminants. So, they end up in drinking water. In groundwater only one compound is detected which is amoxicillin. Once the antibiotics are released into the environment, they undergo a different change in the environment. Sorption to soil and sediments, chemical change such as hydrolysis, photo degradation and biodegradation are some of the environmental fate of antibiotics in the environment ${ }^{12}$. According to different studies $\beta$-lactam and its compounds are susceptible to hydrolysis easily under normal environmental conditions ${ }^{13}$. 

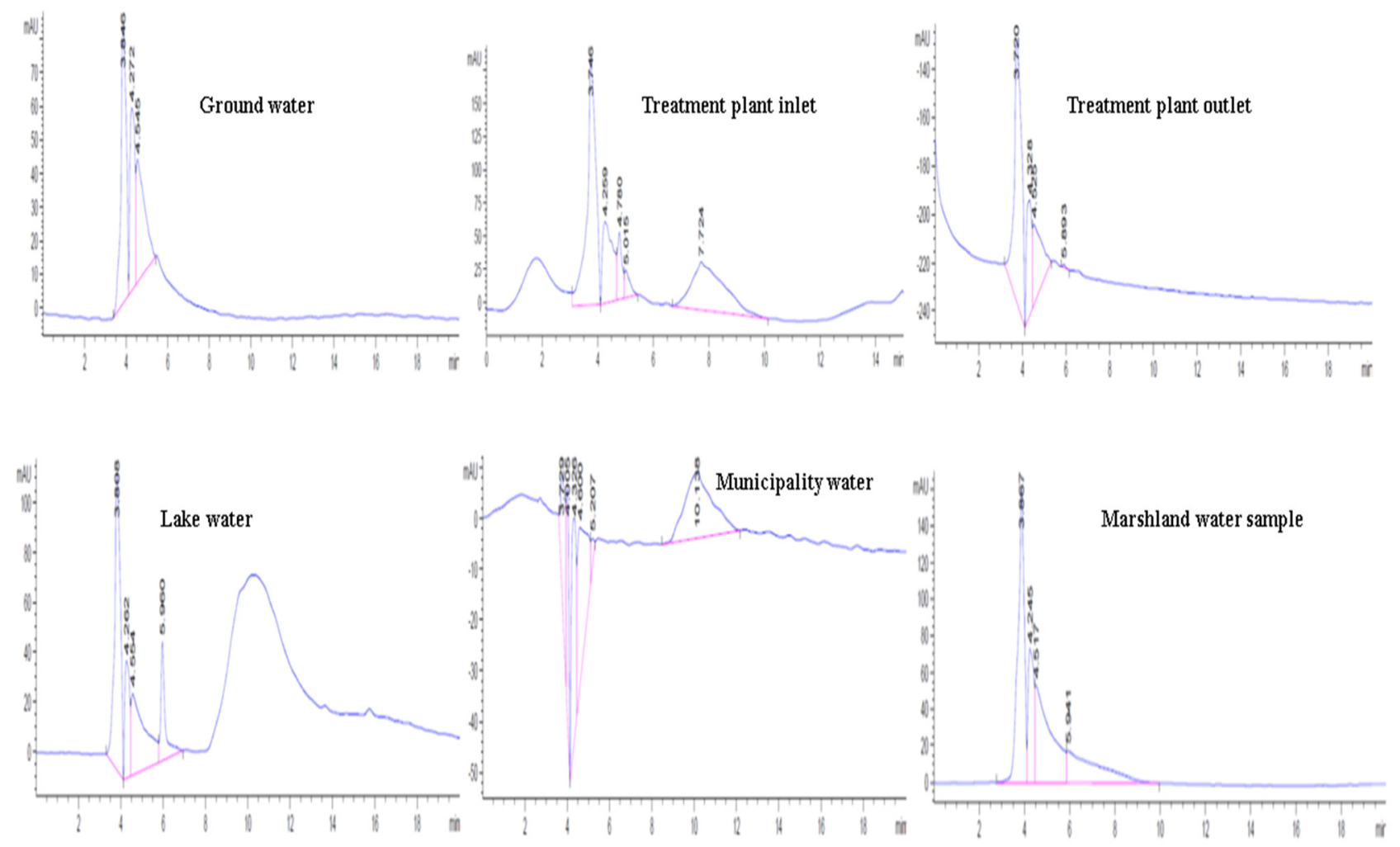

Fig.-3: Chromatogram of Amoxicillin in Various Water Samples

But this result shows the presence of amoxicillin in all the samples which may indicate that these are the fresh sources of antibiotics. Low biodegradability of $\beta$-lactam compounds also reported by another study ${ }^{14}$. Macrolide compounds also susceptible to hydrolysis but not under normal $\mathrm{pH}$ conditions. That may be the reason erythromycin and azithromycin are detected only in some samples.

The chromatogram shows a lot of interferences with some other compounds, so the detection of the target compound is difficult in the samples. The development of a new method is necessary to avoid interferences. This can be done by changing the flow rate, changing the mobile phase and percentage mobile phase in A and $\mathrm{B}$.

\section{CONCLUSION}

The qualitative study indicates that antibiotic residues are present in the water bodies and wastewater treatment plants. This may lead to the development of antibiotic resistance in the environment which may become a critical public health issue. Inappropriate and misuse of antibiotics may increase the threat levels. So, there should be some restrictions in prescribing the antibiotics to patients. The occurrence of antibiotics in the wastewater treatment plant, indicates the need for development of innovative treatment methods, which can remove various kinds of micropollutants. Furthermore, the study is to be required to quantitatively measure the concentration of antibiotics in surface water and wastewater treatment plant. The study can be conducted in a large area with an increase in a number of samples and with different compounds.

\section{REFERENCES}

1. Christian G. Daughton, Thomas A. Ternes, Environmental Health Perspectives, 107, (1999), DOI: 10.1289/ehp.99107s6907.

2. Ching-Hua Huang, Jay E. Renew, Kristen L. Smeby, Karen Pinkston, David L. Sedlak, Journal of Contemporary Water Research and Education, 2, (2001).

3. E. Gracia-Lor, Sancho J. V., R. Serrano, and Hernandez. F, Chemosphere, 87,453(2012), DOI: 10.1016/j.chemosphere.2011.12.025. 
RASĀYAN J. Chem.

Vol. 12 | No. 2 |915 - 920| April - June | 2019

4. Ghazanfar Ali Khan, Bjorn Berglund, Kashif Maqbool Khan, Per-Eric Lindgren, Jerker Fick, PLoS ONE, 8. (2013), DOI: 10.1371/journal.pone.0062712.

5. Mohsen Haidari, Maryam Kazemipour, Bijan Bina, Afshin Ebrahimi, Mehdi Ansari, Mohammad Ghasemian, Mohammad Mehdi Amin, Journal of Environmental and Public Health, (2013), DOI: $10.1155 / 2013 / 351528$.

6. Narendra Nyola and GovindasamyJeyabalan, Indo American Journal of Pharmaceutical Research, 2, (2013).

7. Pei-Ying Hong, Nada Al-Jassim, Mohd Ikram Ansari, Roderick I. Mackie, Antibiotics, 367(2013), DOI: $10.3390 /$ antibiotics2030367.

8. Rahzia Hendricks, Edmund John Pool, Journal of Environmental Science and Health, 289(2012), DOI: $10.1080 / 10934529.2012 .637432$.

9. S. Yang., Cha J. M, Carlson K. H, Journal of Chromatography, 1115, 46(2006), DOI: 10.1016/j.chroma.2006.02.086.1

10. Volmer, D. A, J. P. M. Hui, Rapid Communications in Mass Spectrometry, 12,123(1998), DOI: 10.1002(SICI)1097-0231(199711)11:17<1926:: AID-RCM97>3.0.CO;2-2.

11. Pravin K. Mutiyar, Atul K. Mittal, Desalination and Water Treatment, 51, 6158(2013), DOI: $10.1080 / 19443994.2013 .770199$.

12. Ching-Hua Huang, Jay E. Renew, Kristen L. Smeby, Karen Pinkston, David L. Sedlak, Journal Of Contemporary Water Research and Education, Open Access, 30(2011), DOI:10.1142/9789812799555_0004.

13. J. P. Hou, J. W. Poole, Journal of Pharmaceutical Sciences, 58, 447(1969), DOI:10.1002/jps.2600580412.

14. Al-Almad, A, F. D. Daschner, K. Kümmerer, Archives of Environmental Contamination and Toxicology, 37, 158(1999), DOI: 10.1007/s002449900501.

15. J.Mamatha, N.Devanna, Rasayan Journal of Chemistry, 11(1), 392(2018), DOI:10.7324/RJC.2018.11111931

[RJC-5161/2019] 